\title{
Instabilités de versants de terrils en relation avec l'autocombustion des schistes et charbons résiduels
}

\section{A. MONJOIE C. SCHROEDER}

Laboratoires de Géologie de I'Tngénieur

d'Hydrogéologie et de Prospection géophysique

Sart Tilman B19

B-4000 Liège (Belgique) A.Monjoie@ulg.ac.be Christian.Schroeder@ulg.ac.be
Les terrils de Wallonie font partie du patrimoine de cette région de Belgique. Ils sont parfois exploités pour en extraire le charbon résiduel ou pour servir de matériaux de remblais ou de recouvrement. Ils présentent par ailleurs un indéniable intérêt biologique, paysager, environnemental et même touristique. Les instabilités des terrils se produisent principalement lors de leur édification et leurs causes et mécanismes sont bien identifiés.

Un type d'instabilité moins connu provient de l'autocombustion du charbon résiduel qui, en provoquant des hétérogénéités au sein de la masse du terril, peut dans certaines conditions, conduire à des glissements de terrains, souvent importants, instantanés et sans indices préalables.

Après un bref rappel du phénomène d'autocombustion, le présent article illustre ce type d'instabilité par trois exemples de terrils dont la combustion en cours a conduit à des situations d'instabilité, manifeste ou potentielle. Le papier présente dans chaque cas les résultats des investigations géologiques, hydrogéologiques, géophysiques et géotechniques ainsi que les éventuels calculs de stabilité et solutions retenues.

Mots-clés : terril, stabilité, auto-combustion, glissements, Belgique.

\section{Instabilities of spoil heaps slopes related to the spontaneaous combustion of residual schists and coals}

In Wallonia, spoil heaps are an important element of the landiscape and of the patrimony. They are sometimes worked for recovering the residual coal or as filling material. The spoil heaps instabilities occur mainly during their construction and the causes and mechanisms are well known.

A less frequent instability type comes from the spontaneous combustion of the residual coal which induces some heterogeneity in the spoil heap mass and lead sometimes to instantaneous and more or less important slides. After a short recall of the spontaneous combustion phenomenon, the paper presents three recent case histories of unstable burning spoil heaps. The geological, hydrogeological and geotechnical surveys performed and, in case of need, the stability studies and the proposed solutions.

Key words ; spoil heap, stability, combustion, coal, landslide, Belgium. 


\section{Introduction}

En Wallonie, les bassins charbonniers se situent selon une bande de terrains du Houiller qui traverse le pays d'ouest en est, le long du sillon Sambre et Meuse. Les terrils des charbonnages, édifiés depuis le siècle dernier, font partie du paysage, de l'histoire et du patrimoine de ces régions minières (Fig. 1).

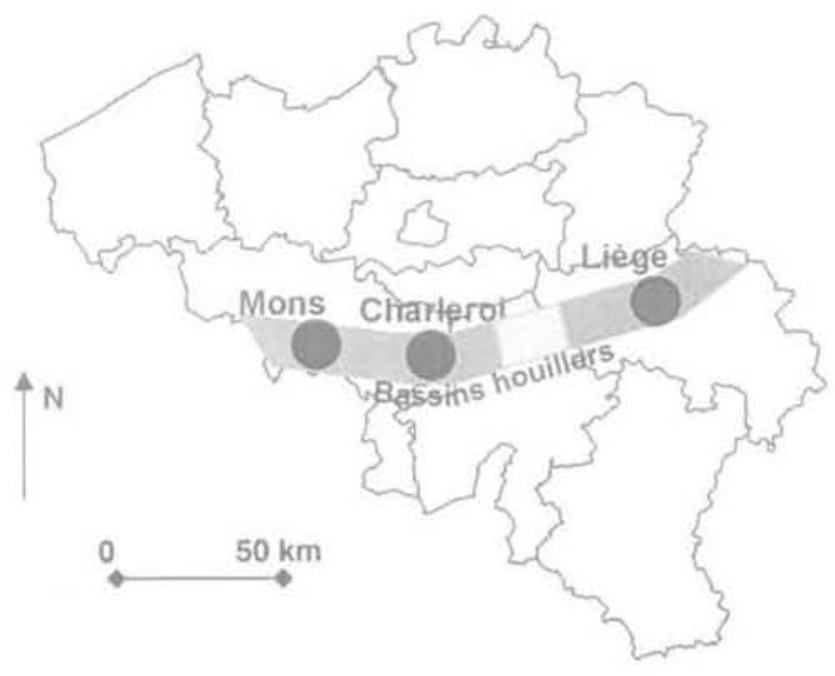

FG.1 Bassins houillers de Wallonie et localisation des études présentées.

Coal basins in Wallonia and location of the case histories.

Bien qu'en Wallonie, les activités extractives soient terminées depuis longtemps (fermeture du dernier charbonnage wallon en 1984), les terrils (parfois orthographiés (c terris n) continuent à faire l'objet d'intérêts divers. Économiquement, le charbon résiduel qu'ils contiennent (de l'ordre d'une dizaine de pourcents en moyenne) a conduit, dans les années 80 , à la création de nombreuses sociétés ayant pour objet la récupération du charbon. Après extraction de celui-ci, les stériles sont généralement remis en place et le terril est «remodelé ». Les autres utilisations industrielles des terrils consistent à les valoriser comme matériaux de remblais et, dans le cas de schistes brûlés (schistes (crouges $»)$, comme matériau de revêtement et autres applications plus « nobles $»$. Plus anecdotiquement, en raison de leurs teneurs en matériaux fins et de leur couleur noire absorbant le rayonnement solaire, les terrils peuvent servir de matériaux d'amendement des sols ou de terrains de vignobles. Au point de vue environnemental (aspects paysager, botanique, zoologique...), et affectif, les terrils sont appréciés des riverains qui, souvent, manifestent une réticence certaine à l'exploitation des terrils de leur voisinage. Dans cet esprit, certains terrils, ont été classés et aménagés comme zones vertes, de promenade ou de loisirs.

\section{2}

\section{Stabilité des terrils}

Lors de leur édification, les terrils peuvent présenter un certain nombre de glissements et autres instabilités bien connues (Marlière, 1950; British National Coal Board, 1970). Elles sont d'abord en relation avec les conditions de dépôts (versage par le haut, sans compactage). En règle générale, cette mise en œuvre pose peu de problèmes particuliers en ce qui concerne le terril sensu stricto, un profil d'équilibre étant rapidement atteint et la compaction des dépôts augmentant naturellement avec le temps, sous leur charge propre mais des instabilités peuvent être dues aux conditions du sous-sol.

Les mouvements des terrains sous-jacents aux terrils surviennent en particulier lorsque le terril pose sur une couche argileuse de relativement faible épaisseur, surmontant un substratum plus rigide (formations du Houiller ou craies par exemple). Les « bourrelets périphériques ») sont caractéristiques de ces instabilités. Celles-ci peuvent survenir rapidement, par rupture, lors de l'édification du terril, causant des dommages importants (Linard de Guertechain, 1939; Schroeder, 1981) ou, le plus souvent, se manifester progressivement et continûment, durant des dizaines d'années, par simple fluage des matériaux argileux sans autre conséquence qu'une légère modification du relief en pied de terril.

Une cause moins connue d'instabilité des terrils est la combustion interne des schistes et charbons résiduels. Le phénomène en tant que tel est connu depuis longtemps ; il est même fait référence à de telles combustions en Angleterre au XVII ${ }^{\circ}$ siècle (Glasser et Bradshaw, 1990). En lui-même, il constitue une source de nuisances diverses telles les fumées sulfureuses. De plus, en provoquant des hétérogénéités au sein de la masse du terril, la combustion des schistes et charbons peut dans certaines conditions, conduire à des glissements de terrains, parfois fort importants.

Après avoir rappelé le phénomène d'autocombustion, le présent article illustre ce type d'instabilité par trois exemples de combustions de terrils ayant conduit à des situations d'instabilité, manifeste ou potentielle. Ces exemples sont situés dans les régions de Liège, Charleroi et Mons.

\section{3}

\section{Autocombustion des schistes et charbons résiduels}

Les schistes d'exploitation du charbon habituellement déposés en terrils contiennent encore de l'ordre de 5 à $15 \%$ de charbon, ce pourcentage dépendant de l'âge du terril, les terrils les plus anciens (ceux édifiés avant les techniques de flottation) étant en règle générale ceux qui renferment le plus de charbon résiduel. D'autre part, les schistes renferment parfois une quantité non négligeable de sulfure de fer (pyrite, marcassite) pouvant donner en présence d'eau et d'oxygène et dans certaines conditions, une réaction exothermique. Lorsque le terril présente un volume suffisant pour atteindre une $\alpha$ masse critique $)$ (ce qui signifie une hauteur de terril supérieure à 5 mètres au moins) et est peu compact, ce qui permet une circulation d'air et d'eau, le phénomène d'autocombustion peut se produire et dans certains cas, la totalité du charbon résiduel peut brûler, l'extinction des terrils en feu n'étant pratiquement pas possible du point de vue économique (Hebley, 1949). 
Ces combustions présentent de réels dangers lorsqu'elles sont en cours. Outre les effets de la chaleur, de nombreux accidents peuvent être provoqués par les combustions des terrils. C'est ainsi que :

- des gaz nocifs sont émis lors de la combustion. Le monoxyde de carbone est le plus dangereux, les fumées sulfureuses étant irritantes et toxiques à doses élevées ;

- la combustion des schistes charbonneux conduit à la formation de cavités sous la surface ( $\propto$ marmites $)$ ou " chaudrons m), laissant en surface une croûte qui peut s'effondrer sous le poids d'une personne;

- la vapeur d'eau, dégagée lors de pluies ou d'arrosages du terril, peut former du water-gas, mélange de monoxyde de carbone et d'hydrogène qui, lorsqu'il est en certaines proportions avec de l'air, donne un mélange explosif. L'explosion, lorsqu'elle se produit. par exemple lorsqu'une excavatrice perce une poche remplie de ce gaz, donne une dispersion de matériaux brûlants qui a par le passé causé le décès de plusieurs personnes (ce fut le cas en Belgique et en Grande-Bretagne) :

- au cours d'excavation de terrils en combustion, des glissements peuvent entrainer des nuages de poussières charbonneuses qui peuvent exploser au contact des matières incandescentes.

Les terrils brûlés (terrils rouges) fournissent d'excellents matériaux de remblais et sont recherchés pour des usages particuliers (allées, courts de tennis, etc.). Pour cette raison, par le passé, il a parfois été mis volontairement feu aux terrils (Harrington et Heast. 1949).

\section{1}

\section{Mécanismes de l'autocombustion}

La combustion des charbons résiduels est principalement due à une réaction exothermique de l'oxydation des pyrites mais des cas d'inflamation de charbons "purs » par ( simple » oxydation du charbon semblent être rencontrés (Harrington et Heast, 1949; Carras et Leventhal, 2000).

En présence de sulfures, cette réaction complexe est généralement présentée comme composée des phases suivantes :

1) oxydation des pyrites (et surtout des marcassites et pyrrhotine) qui donne du sulfate ferreux et de l'acide sulfurique :

$$
2 \mathrm{FeS}_{2}+7 \mathrm{O}_{2}+2 \mathrm{H}_{2} \mathrm{O} \rightarrow 2 \mathrm{FeSO}_{4}+2 \mathrm{H}_{2} \mathrm{SO}_{4}
$$

2) réaction bactérienne (bactéries aérobies autotrophes du groupe des ferrobaciles - thiobaciles, nécessitant un environnement à $\mathrm{pH}<4,5$, avec un $\mathrm{pH}$ optimum de 2,2) avec le sulfate ferreux (qui autrement formerait une couche protectrice) permettant la formation de sulfate ferrique :

$$
\begin{gathered}
\text { 4 } \mathrm{FeSO}_{4}+\mathrm{O}_{2}+2 \mathrm{H}_{2} \mathrm{SO}_{4}+\text { activité bactérienne } \\
\qquad 2 \mathrm{Fe}_{2}\left(\mathrm{SO}_{4}\right)_{3}+2 \mathrm{H}_{2} \mathrm{O}
\end{gathered}
$$

3) nouvelle réaction avec la pyrite et réduction du sulfate ferrique :

$$
7 \mathrm{Fe}_{2}\left(\mathrm{SO}_{4}\right)_{3}+\mathrm{FeS}_{2}+8 \mathrm{H}_{2} \mathrm{O} \rightarrow 15 \mathrm{FeSO}_{4}+8 \mathrm{H}_{2} \mathrm{SO}_{4}
$$
duire:

De plus, les réactions suivantes peuvent se pro-
4) formation de limonite et d'acide :

$$
\mathrm{FeSO}_{4}+2 \mathrm{H}_{2} \mathrm{O} \rightarrow \mathrm{Fe}(\mathrm{OH})_{2}+\mathrm{H}_{2} \mathrm{SO}_{4}
$$

5) formation de jarosite $\left(\mathrm{KHFe}_{3}\left(\mathrm{SO}_{4}\right)_{2} \cdot(\mathrm{OH})_{6}\right)$

a) $3 \mathrm{Fe}_{2}\left(\mathrm{SO}_{4}\right)_{3}+12 \mathrm{H}_{2} \mathrm{O} \rightarrow 2 \mathrm{HFe}_{3}\left(\mathrm{SO}_{4}\right)_{2} \cdot(\mathrm{OH})_{6}+$ $5 \mathrm{H}_{2} \mathrm{SO}_{4}$

b) $\mathrm{HFe}_{3}\left(\mathrm{SO}_{4}\right)_{2} \cdot(\mathrm{OH})_{6}+\mathrm{K}$ (argile ou micas) $\rightarrow$ (en environnement acide)

$\mathrm{KHFe}_{3}\left(\mathrm{SO}_{4}\right)_{2} \cdot(\mathrm{OH})_{6}+\mathrm{K}$ (argile ou micas) $+\mathrm{H}^{*}$

Dans certaines conditions, il peut y avoir formation de mélantérite $\left(\mathrm{FeSO}_{4} \cdot 7 \mathrm{H}_{2} \mathrm{O}\right)$.

En fait, ces réactions sont compliquées par la présence de différentes formes minéralogiques des sulfures de fer pouvant réagir les unes avec les autres et catalyser les réactions. Le résultat final est de l'acide sulfurique et de la chaleur. Comme la réaction d'oxy. dation est accélérée par une augmentation de la température, dès que la balance de l'échange thermique avec l'environnement produit une élévation de la température du terril, la réaction s'accélère et peut donner lieu à l'auto-inflammation de charbon résiduel.

La réaction nécessite donc de l'oxygène (terril peu compact) et de l'eau pour produire la chaleur nécessaire au départ de la combustion. Elle est favorisée par la présence de sulfures, surtout la marcassite et la pyrrhotine qui catalysent l'oxydation de la pyrite.

La combustion s'initie la plupart du temps au coeur du terril, dans une zone où les échanges thermiques avec l'environnement sont limités, et progresse vers le haut (effet de cheminée) et vers l'extérieur. Bien entendu, la propagation du feu est fonction des répartitions des combustibles et il n'est pratiquement pas possible de prévoir les directions dans lesquelles les combustions vont se propager. Les températures atteintes au sein d'un terril en combustion peuvent atteindre et dépasser les $400^{\circ} \mathrm{C}$, voire davantage (Carras et Leventhal, 2000). On a signalé localement $1200^{\circ}$.

\section{2}

\section{Instabilités des terrils en combustion}

Pratiquement toutes les ruptures \& naturelles s dans les versants de terrils en combustion surviennent lors de fortes pluies. La pénétration d'eau dans le massif en ignition agit d'une manière complexe et fait intervenir simultanément plusieurs éléments :

D'une part, les précipitations ont un effet direct en raison de

- l'augmentation du poids volumique des matériaux non brûlés (superficiels) qui se saturent en eau ;

- la saturation de ces matériaux, initialement non saturés, entraîne une diminution de la suction et, partant, une diminution de la résistance au cisaillement (Fredlund et Rahardjo, 1993) ;

- l'instauration d'une nappe temporaire, proche de la surface, provoquant une augmentation des pressions interstitielles ;

- l'effet du mouvement vers l'aval de cette nappe, créant des forces déstabilisantes.

Par ailleurs, les eaux de pluie pénétrant dans le massif en combustion se vaporisent et induisent des pressions de vapeurs agissant sur la face inférieure de la partie non brûlée et de ce fait déstabilisent celle-ci. Cette pression de vapeur se manifeste en partie 
médiane car en dessous, la nappe logée dans les flancs du terril empêche la propagation de la vapeur vers l'extérieur à partir du moment où la pression de la colonne d'eau excède la pression de la vapeur. En partie haute du terril, la vapeur produite peut aisément s'échapper par le chemin préférentiel (« cheminée n) suivi par les gaz de combustion. La zone critique se situe donc généralement vers la mi-hauteur du terril.

La surface de discontinuité séparant, au sein du terril, les schistes brûlés des schistes non brûlés constitue en général la surface de glissement en raison de la différence de caractéristiques mécaniques des schistes brûlés. Ceux-ci sont plus résistants que les schistes "noirs », du fait de la " cuisson » prolongée du matériau et de l'augmentation de compacité (départ de la matière la plus légère, le charbon, et des matières organiques, retrait après " cuisson $»)$.

Le schéma de la figure 2 illustre ce mécanisme. Sur cette figure, on peut voir que le matériau après glissement s'étend sur une assez grande longueur. Le glissement, même s'il a lieu le long d'une surface raisonnablement cylindrique (ou plutôt sphérique), n'est pas un glissement rotationnel classique. En raison de la teneur en eau élevée des schistes, le mouvement de la masse glissée s'apparente plutôt à des coulées boueuses qui se propagent sur de grandes distances.

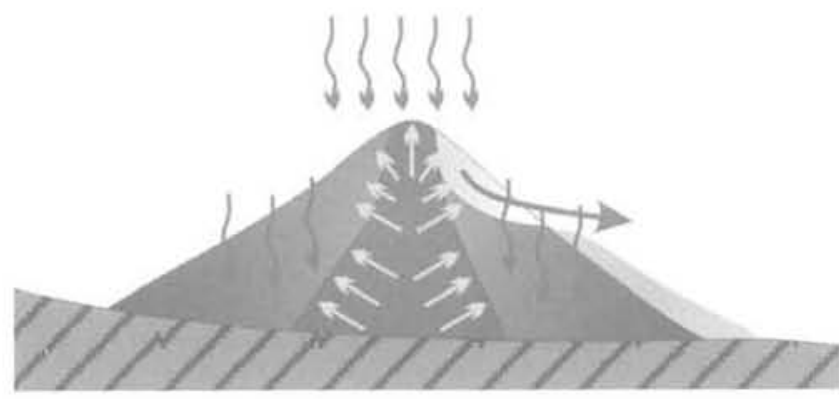

FiG.2 Mécanisme du glissement dans un terril en combustion.

Mechanism of landslides in a burning spoil heap.

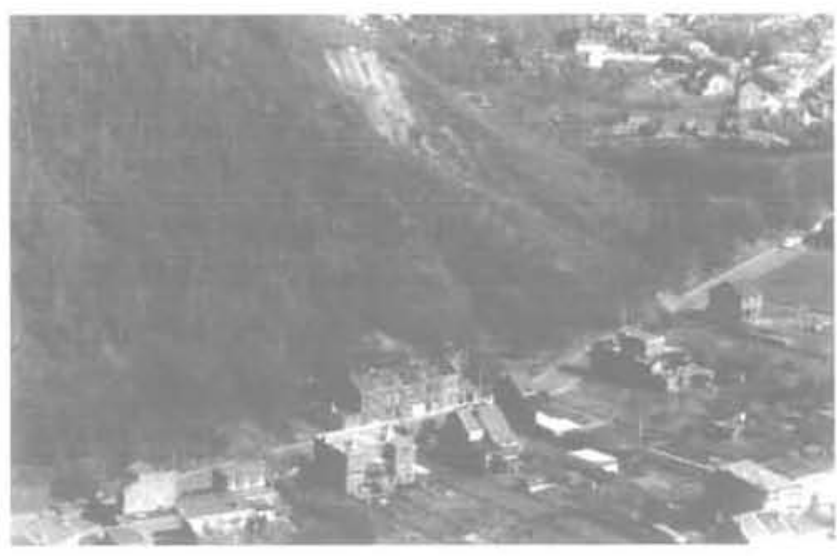

\#G.3 Terril de la petite Bacnure à Herstal : vue générale du glissement.

Petite Bacnure spoil heap in Herstal : General view of the landsilide.

Les tomographies électriques ont permis de faire la différence entre les schistes sains et les schistes brûlés ou en combustion, sur la base des résistivités électriques. Ces résistivités varient suivant la nature du terrain. Les valeurs généralement observées sont
SE

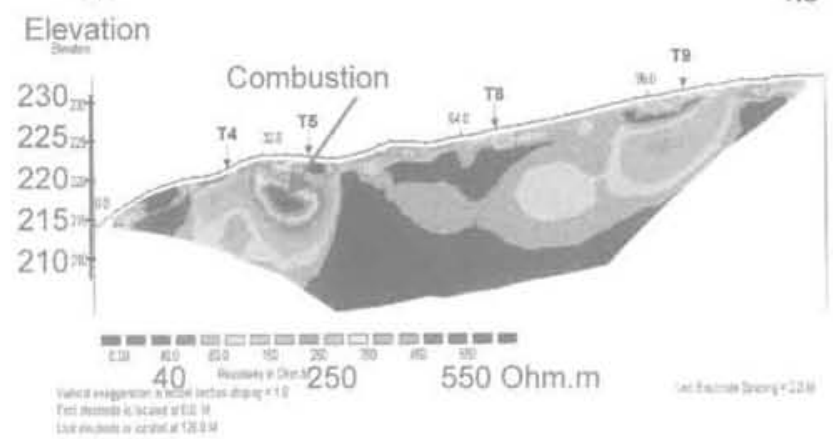

FiG.4 Tomographie électrique d'une zone partiellement en combustion, en tête du glissement.

Electrical tomography in a partially burning zone, at the top of the slide.

\section{4}

\section{Terril de la petite Bacnure à Herstal (région liégeoise)}

Le glissement du terril de la petite Bacnure illustre parfaitement le mécanisme décrit plus haut. La figure 3 montre la surface de glissement, rouge en haut du talus et la masse glissée, d'un volume total glissé de l'ordre de $2000 \mathrm{~m}^{3}$, qui s'est arrêtée à quelques mètres de la maison en face. Ce glissement est purement local mais peut se répéter ailleurs. Par contre, la stabilité classique d'ensemble du terril, posant directement sur le socle houiller, n'a jamais été mise en cause.

Après le glissement, compte tenu de l'urgence et des difficultés d'accès, une investigation géophysique par tomographies électriques et sondages sismiques a été réalisée sur l'ensemble du terril. Les sondages sismiques (réfraction) ont pour objet de déterminer les épaisseurs des zones superficielles, moins compactes, susceptibles de glisser.

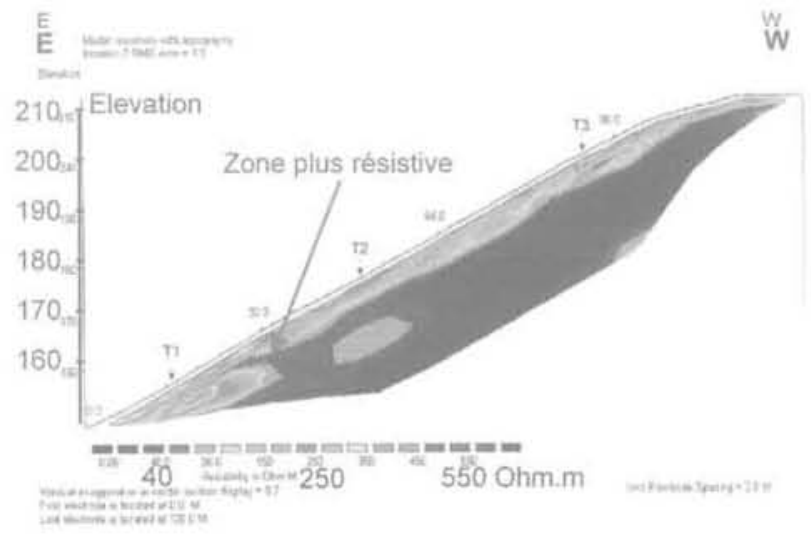

FIG.5 Tomographie électrique d'une zone pratiquement exempte de combustion. Electrical tomography in a zone practically without combustion. 
- schistes non brûlés ( ( noirs », ( sains ») : 20 à $120 \Omega . m$; - schistes gréseux ou grès : entre 100 et $300 \Omega$.m :

- schistes brûlés ( $($ rouges ») : plus de 200 à $300 \Omega$.m: - schistes chauds : plus de $400 \Omega$.m et pouvant dépasser $1000 \Omega$.m.

Les figures 4 et 5 donnent deux exemples de tomographies électriques, l'une dans une zone en combustion (crête du terril en haut du glissement), l'autre dans une zone exempte de combustion (versant opposé à celui du glissement). Sur ces profils, les couleurs claires (rouges) correspondent aux résistivités élevées caractéristiques des schistes brûlés ou chauds.

Sur le profil tomographique de la figure 5, la zone claire (rouge) en pied de terril est peut-être due à un remblai localement plus gréseux qui normalement s'accumule en pied de terril.

Les observations faites par prospection géophysique ont été complétées par un lever topographique détaillé et par une thermographie en photos aériennes infrarouges qui ont permis de confirmer l'étendue des zones en combustion. La figure 6 donne un exemple de thermographie de la zone du glissement. On voit nettement la tache blanche au sommet du glissement, caractéristique d'une zone chaude, correspondant au point T5 de la figure 4.

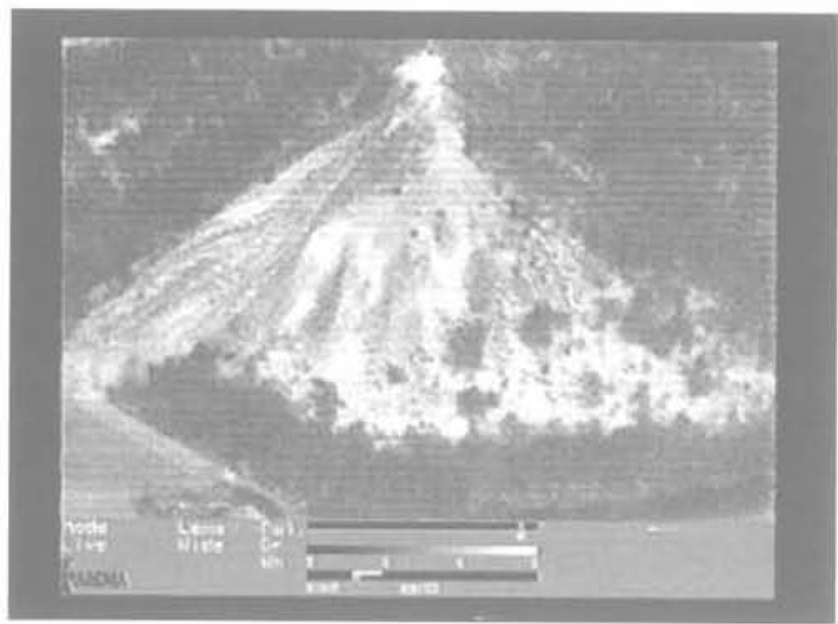

FG. 6 Thermographie infrarouge de la zone du glissement.

Infrared thermography of the landsidie area.

La campagne de reconnaissances a finalement permis de déterminer les zones les plus instables, c'est-à-dire celles à pente raide présentant des zones brûlées en profondeur et des épaisseurs importantes de terrains peu compacts.

\section{5}

\section{Terril Saint-Charles à Ransart (région carolorégienne)}

Le terril Saint Charles, d'une hauteur de quelque $60 \mathrm{~m}$, couvrant environ 6 hectares, est en feu depuis 1994. La figure 7 montre une vue générale du terril avec sur pratiquement toute la surface du terril, des troncs d'arbres calcinés. Le terril pose sur les terrains du Houiller et sa stabilité classique d'ensemble ne pose pas de problème.

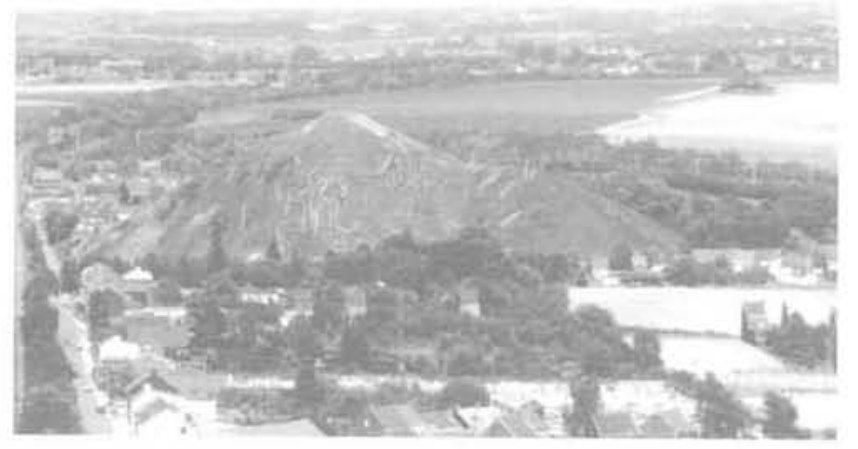

FlG.7 Terril Saint-Charles à Ransart. Vue d'ensemble.

St Charles spoil heap in Ransart, General view.

Actuellement, le terril est toujours en combustion assez intense, combustion atypique en ce sens qu'au lieu de se propager du centre du terril vers l'extérieur, elle progresse de l'extérieur vers l'intérieur. La raison en est que la combustion des schistes a été initialisée par un feu extérieur au terril. En quelques années, la combustion s'est propagée sur pratiquement toute la surface du terril puis a progressé vers l'intérieur de celui-ci. Les premières zones brûlées se revégétalisent progressivement, ainsi qu'on peut le voir sur le côté gauche de la figure 7. La figure 8 montre deux aspects caractéristiques de cette combustion. La combustion
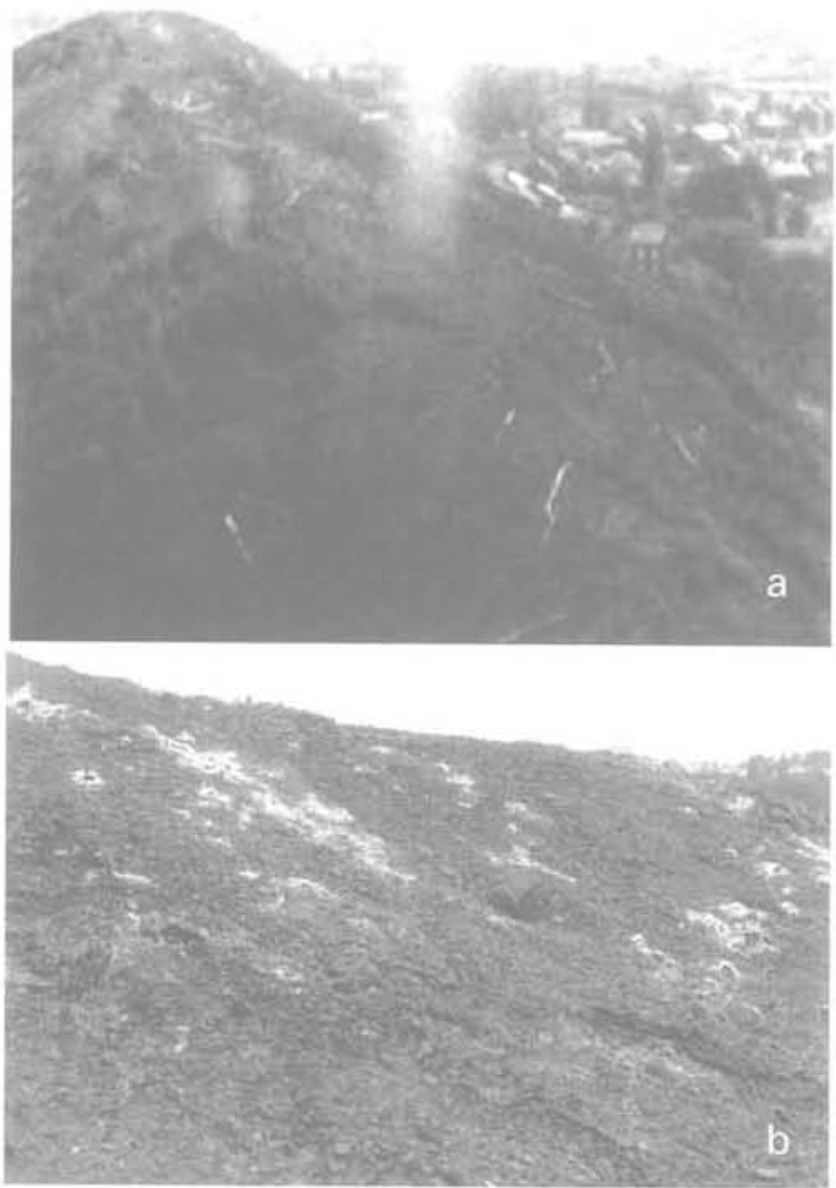

EG.8 Terril Saint-Charles: (a) colonne de vapeur; (b) cristallisations de soufre. St Charles spoil heap : (a) vapor column : (b) sulphur cristallysation. 
intense (la surface du terril est localement brûlante) vaporise l'eau du terril et, même par temps relativement sec, on peut observer des colonnes de vapeur. D'autre part, le dégagement de $\mathrm{H}_{2} \mathrm{~S}$ et autres gaz provoque des efflorescences de soufre en de nombreux endroits.

Du fait de cette disposition, le mécanisme de glissement tel qu'il a été décrit au terril de la Petite Bacnure à Herstal ne s'applique plus exactement. En revanche, la perte de compacité des couches extérieures en réduit la résistance et, surtout, la progression du feu à l'intérieur du terril crée des quantités de gaz non négligeables qui font courir le risque d'une explosion de water-gas.

Les investigations du terril ont été menées à la demande de la ville de Charleroi. L'urgence, les conditions dangereuses (terril en combustion intense) et les difficultés d'accès ont conduit à effectuer les reconnaissances par prospections géophysiques et télédétection. Les reconnaissances ont comporté des tomographies électriques, des sondages sismique réfraction, une thermographie infrarouge aérienne ainsi qu'un suivi topographique.

Les figures 9 à 12 donnent l'implantation des profils sismiques et des tomographies électriques, répartis sur le pourtour du terril et des exemples de profils sismiques et électriques. Les profils électriques montrent les zones brûlées ou en combustion, les profils sismiques, les profondeurs des terrains déconsolidés par

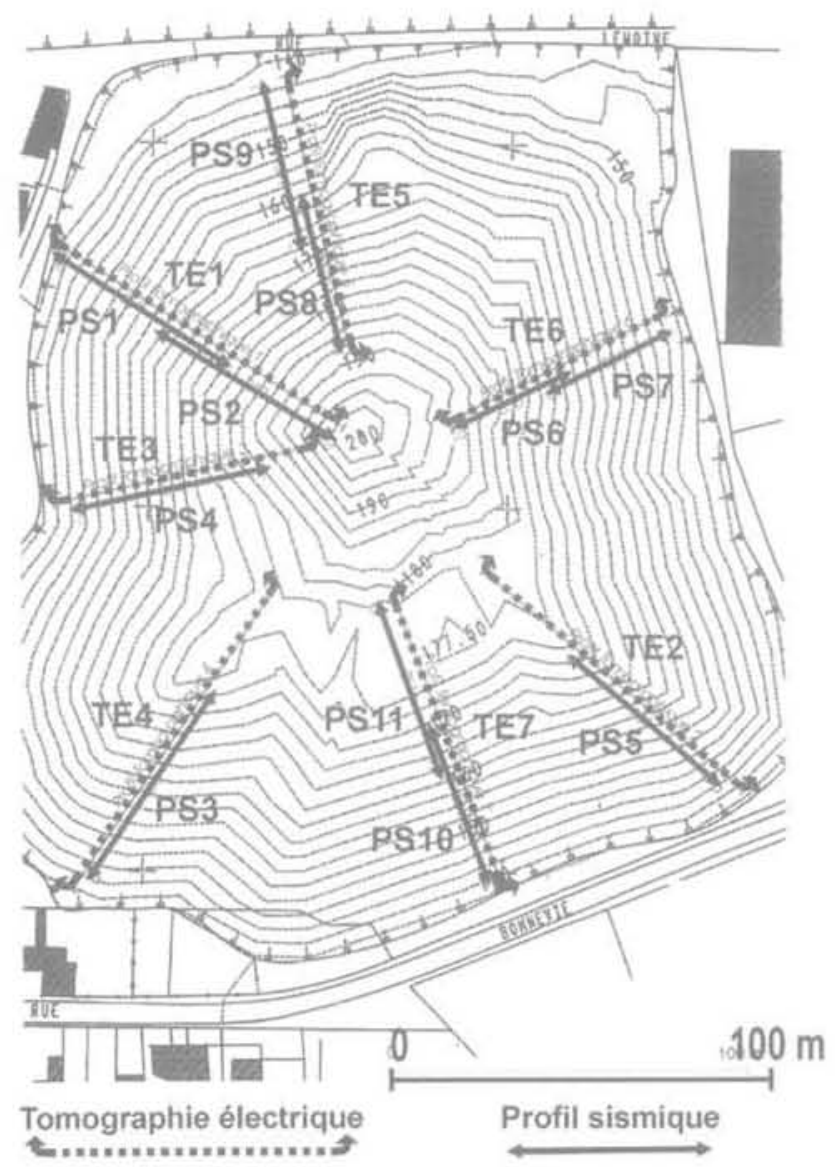

FIG.9 Terril Saint-Charles : implantation des reconnaissances.

St Charles spoil heap : Location of surveys.

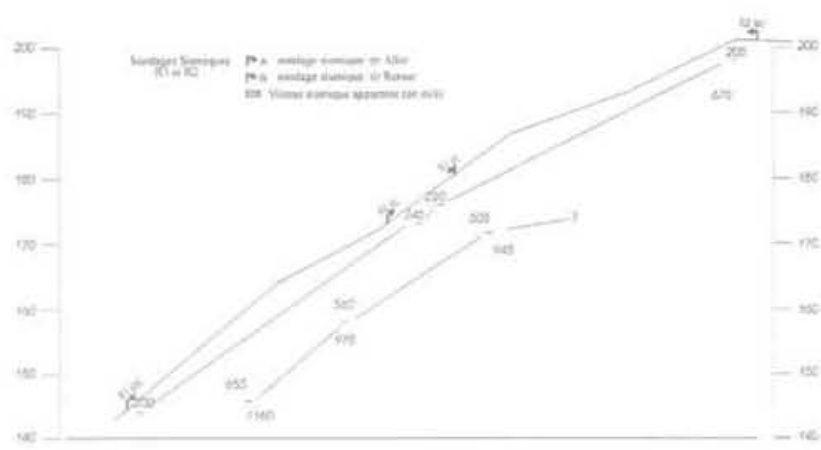

FiG.10 Terril Saint-Charles: exemple de profils sismiques - S1 et S2,

St Charles spoil heap : Example of seismic profiles - S1 and S2.

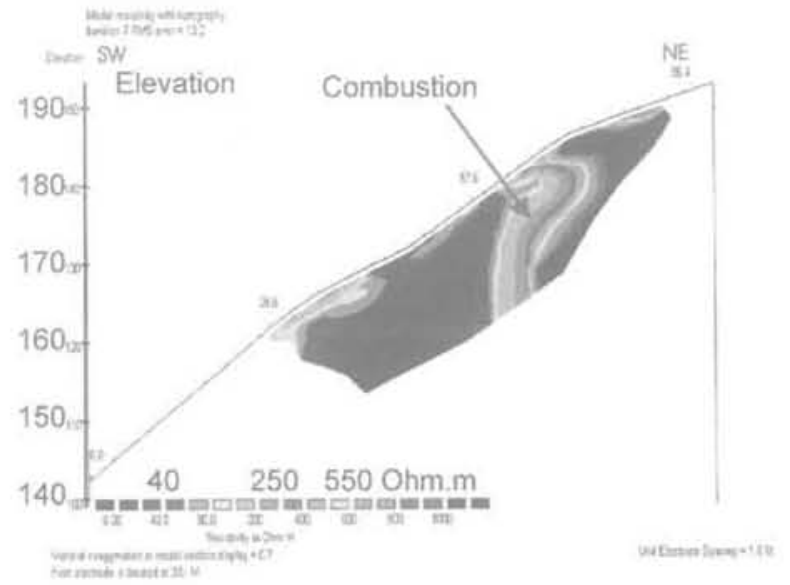

FG. 11 Profil de tomographie électrique correspondant aux profils sismiques de la figure 10.

La partie haute du profil montre de la combustion.

Electrical tomography corresponding to the sesimic profiles of figure 10. At the top, some combustion is pointed out.

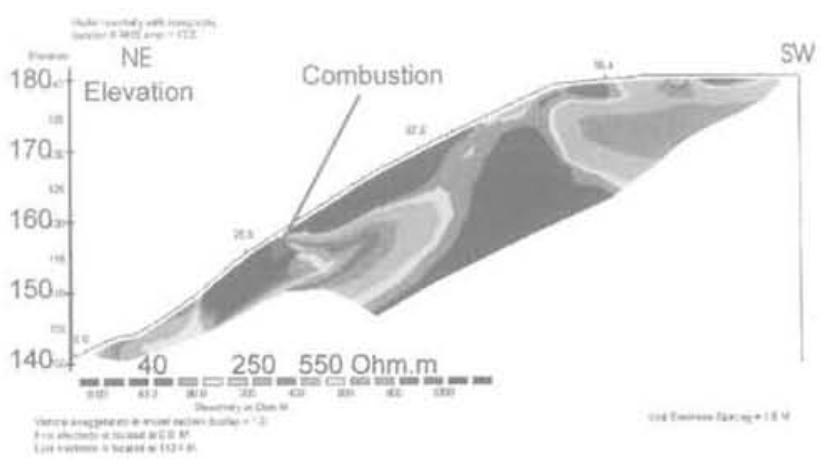

Autre exemple de profil de tomographie électrique - zone chaude en pied de terril. Another example of electrical tomography - hot zone at the toe of the spoil heap. 
la combustion, caractérisés par des vitesses sismiques très lentes, de l'ordre de $200 \mathrm{~m} / \mathrm{s}$, la partie « saine » du terril présentant des vitesses sismiques de l'ordre de $1000 \mathrm{~m} / \mathrm{s}$.

Les figures 13 et 14 montrent la même vue aérienne du terril, l'une étant en couleurs naturelles, l'autre en infrarouges. On note la forte chaleur qui se dégage au sommet du terril (effet cheminée) et la répartition des zones en combustion, avec intensité maximum sur les arêtes convexes.

Les reconnaissances effectuées ont permis de délimiter les zones où les probabilités d'instabilité étaient les plus fortes, zones présentant les plus grands risques d'explosion qui pourrait se produire au cas où un glissement mettrait brutalement en contact avec l'air les masses incandescentes de l'intérieur du terril. Le périmètre de " dangerosité ") ayant été défini, la ville de Charleroi a racheté aux propriétaires les immeubles menacés et a facilité leur relogement. Actuellement, le terril poursuit sa combustion et fait l'objet d'un suivi continu.

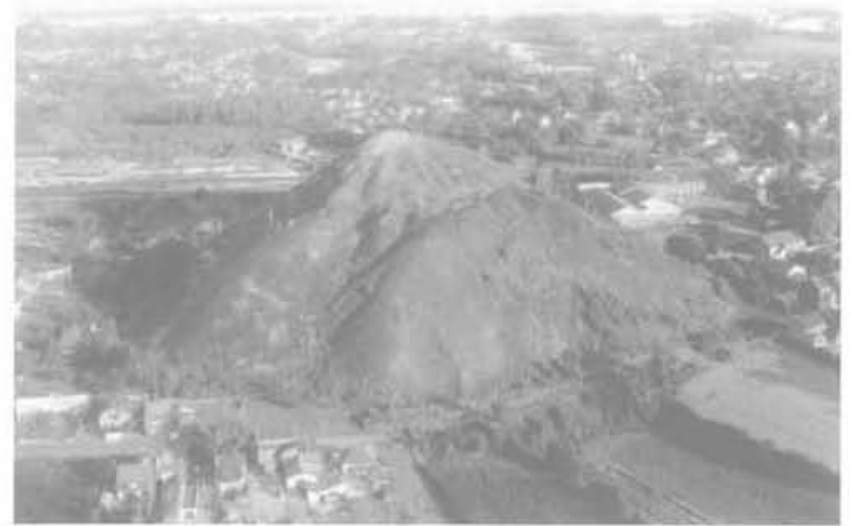

FG. 13 Vue aérienne du terril Saint-Charles. View of the St Charles spoil heap.

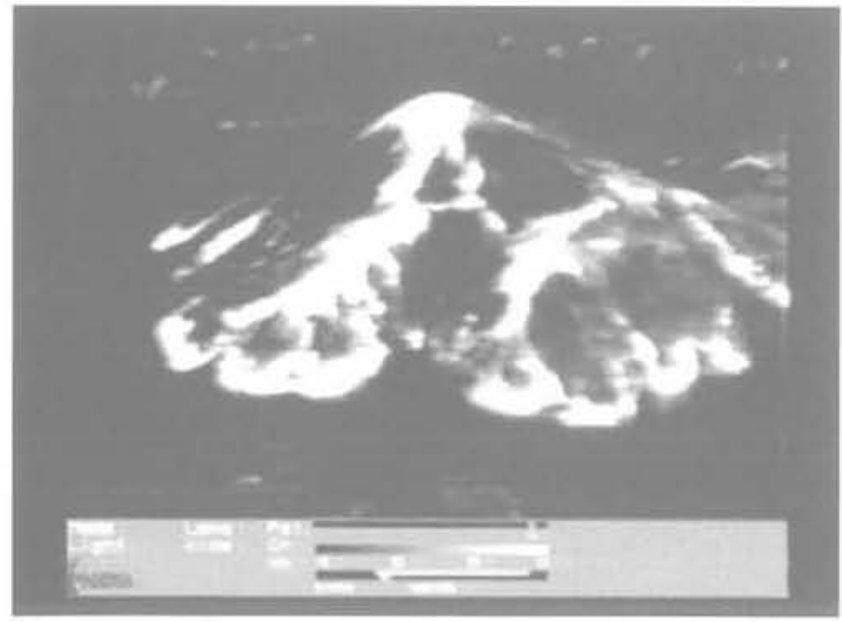

FIG. 14 Thermographie infrarouge du terril SaintCharles.

La vue correspond exactement à celle de la figure 13 .

Infrared thermography of the Saint Charles spoil heap.

The view corresponds exactly to the view of figure 13.

\section{Terril de l'Héribus à Cuesmes (région montoise)}

La vue aérienne de la figure 15 montre le terril de l'Héribus, situé au Sud de la ville de Mons, visible en arrière-plan. Le terril de l'Héribus est haut de $75 \mathrm{~m}$ et s'étend sur quelque 16 hectares. Ce terril présente un intérêt particulier, car il est à la fois le siége de problèmes d'instabilités liées à des combustions internes mais aussi de glissements liés à la nature des terrains de fondation. Le terril date du début du siècle et a été

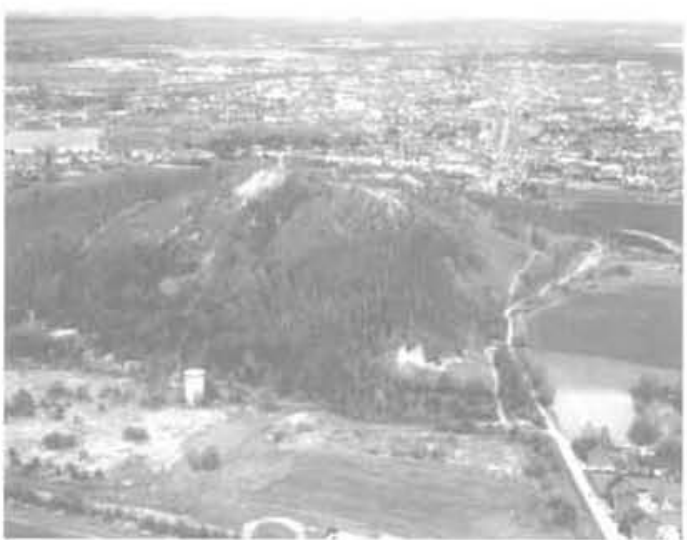

FG. 15 Terril de l'Héribus : vue générale. Héribus spoil heap : General view.

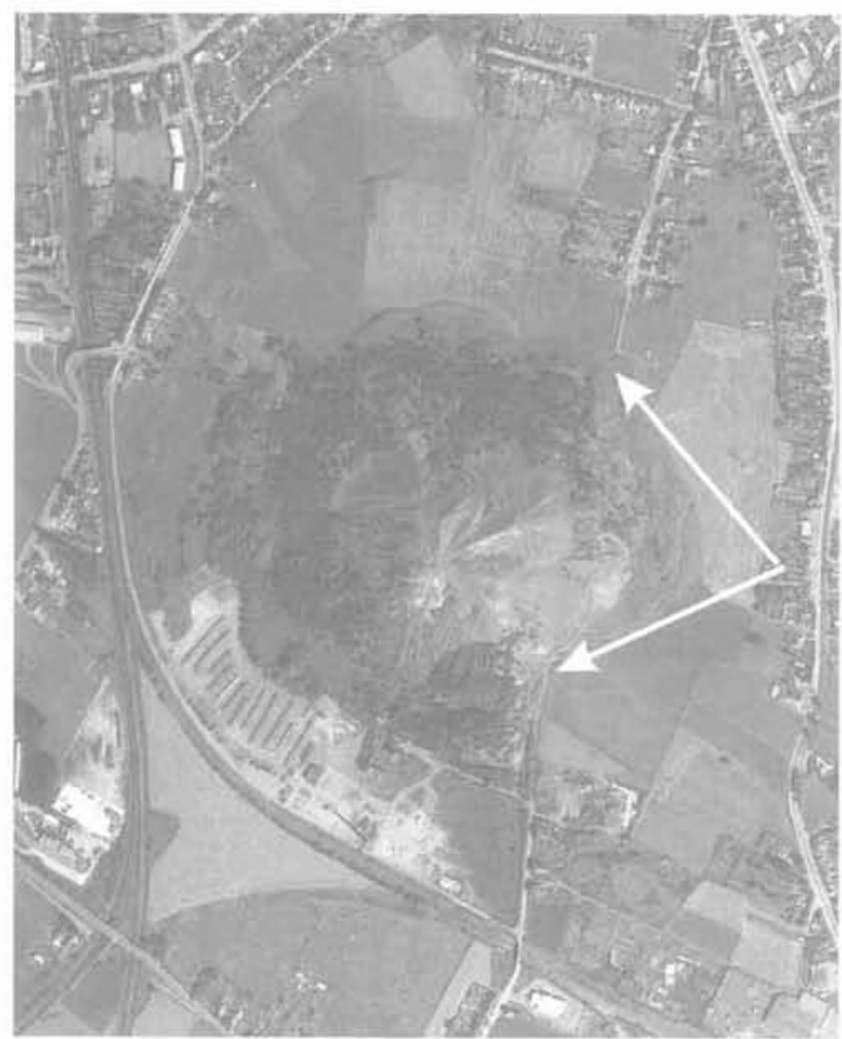

FG.16 Vue aérienne du terril de I'Héribus et de la coupure de la route de Bavay.

Aerial view of the Heribus spoil heap and of the cutting of the Bavay road. 
arrêté assez récemment, le charbonnage de l'Héribus ayant cessé son activité depuis moins de trente ans. Les pentes du terril sont relativement douces en pied de terril $\left(10^{\circ}\right)$, plus redressées vers le sommet $\left(25^{\circ}\right.$ à $\left.30^{\circ}\right)$ Actuellement, le sommet du terril présente des zones en combustion (schistes rouges, quelques fumerolles et léger dégagement de chaleur), visibles sur la figure 15.

Il y a quelques années, un aménagement du terril a été réalisé par les soins de la Ville de Mons (escaliers, chemins, bancs, table d'orientation au sommet, etc.). I constitue encore actuellement un lieu de promenade assez fréquenté.

Au point de vue géologique, le terril de l'Héribus repose sur des formations limoneuses quaternaires épaisses d'une dizaine de mètres. Sous celles-ci, dans les deux tiers Sud du site, on rencontre quelques mètres du complexe argileux yprésien surmontant 20 à 30 mètres du complexe sableux landénien, ce dernier reposant sur les craies et marnes du Crétacé. Le toit des formations du Secondaire a un pendage nord/nord-est Au nord du site, une formation de sables yprésiens (ou paniséliens) s'intercale entre le limon quaternaire et l'argile yprésienne. En ce qui concerne les tassements miniers, la carte géotechnique renseigne des affaissements de l'ordre de $2,50 \mathrm{~m}$ sous le terril et jusqu'à $5,00 \mathrm{~m}$ plus à l'ouest, valeurs confirmées par l'analyse des cartes topographiques réalisées à différentes époques.

En ce qui concerne la stabilité, dès le début de sa réalisation, le terril a présenté deux zones d'instabilité provoquant des bourrelets de pied : au nord (où la situation semble stabilisée) et surtout à l'est où le glissement a coupé la route (chemin de Bavay) passant à cet endroit. La photo aérienne (datant d'une vingtaine d'années, cf. figure 16) montre le déplacement du terril sur le tracé de la route. La partie Sud du terril paraît stable, hormis, très localement.

Deux glissements ont affecté le terril au milieu des années 90 . Ils sont désignés dans la suite par les termes " glissement Est $»$ et « glissement Ouest ». Ils se présentent comme suit. La figure 17 montre les zones d'instabilité et les phènomènes associés (fissures, bourrelets, etc.).

\section{1}

\section{Glissement Est}

En 1994, un glissement très important a affecté le flanc Est du terril. Ce glissement s'est produit assez lentement et, semble-t-il, de manière continue. Il se manifeste par:

- en tête du glissement et quasi au sommet du terril, une niche d'arrachement de près de $7 \mathrm{~m}$ de haut, témoignant d'un mouvement général de rotation d'environ $5^{\circ}$;

- en pied de glissement, d'importants bourrelets situés à la base du terril.

Sur le flanc du terril affecté par le glissement, on note de nombreuses fissures ouvertes avec petits déplacements verticaux ainsi qu'une série de nombreux décrochements orientés est-ouest au pied de ce flanc, sur le chemin et sur le replat en contrebas de ce dernier.

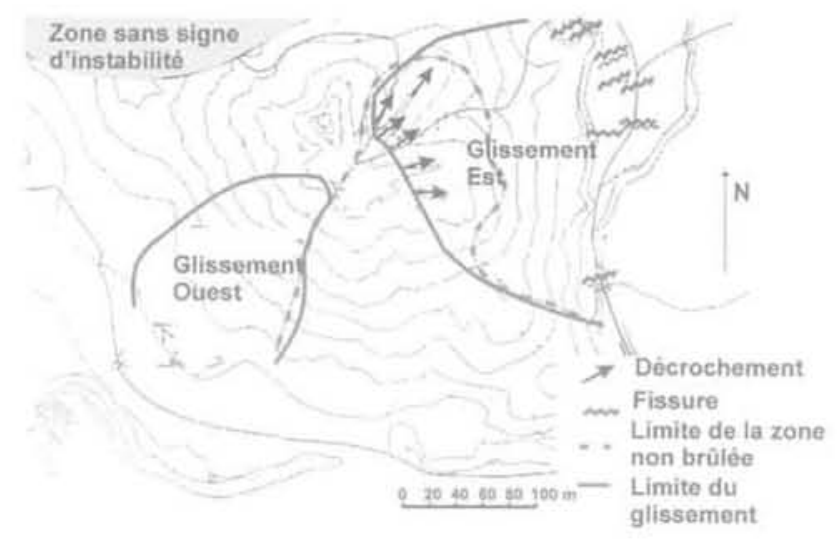

Fig. i7 Terril de l'Héribus : observations des phénomènes d'instabilité.

Héribus spoil heap: Location of the observed instabilities.

\section{Glissement Ouest}

En été 1992, après (ou au cours) un violent orage, un brutal décrochement au sommet du flanc Ouest a provoqué le déplacement rapide d'une masse de terril qui s'est éboulée en contrebas, renversant les arbres et provoquant un mouvement du pied du terril se traduisant par de nombreuses fissures ouvertes et quelques bourrelets supplémentaires en pied. En tête du glissement, la paroi qui subsiste actuellement (faisant apparaitre le schiste brûlé) a une pente assez raide et sur le replat, au-dessus du glissement, on remarque des fissures (amorces de glissement) à une dizaine de mètres de la table d'orientation. Près du sommet, subsiste une zone en combustion avec fumerolles.

Les risques évidents d'instabilité du glissement Ouest et la dégradation continue par le glissement Est ont conduit la ville de Mons à faire effectuer une étude détaillée des glissements. Ces études ont comporté des reconnaissances in situ et des essais de laboratoire dont les résultats ont été utilisés pour les calculs de stabilité. En même temps qu'une inspection détaillée du terrain (sur le site et sur photos aériennes) avec

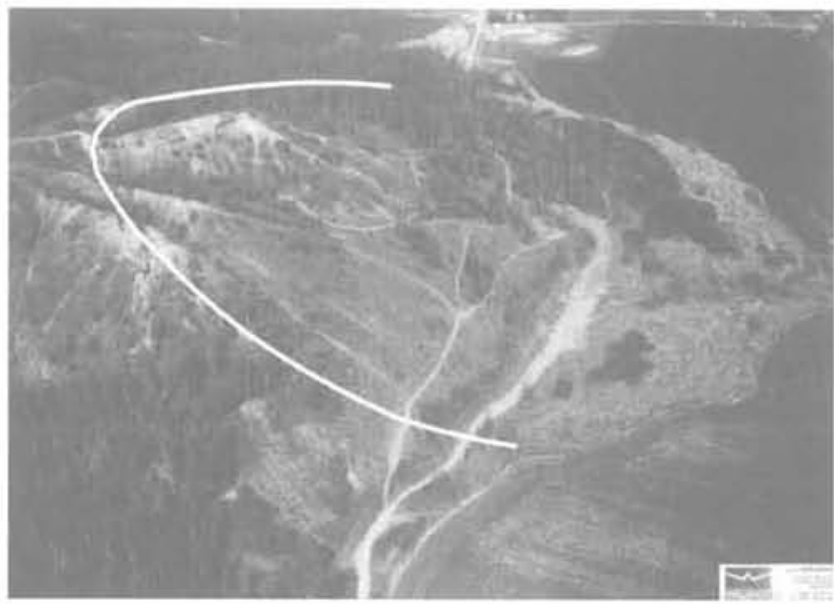

FIG,18 Glissement Est : avancée du bourrelet de pied, escarpement de tête. East slide : toe-head scarp. 


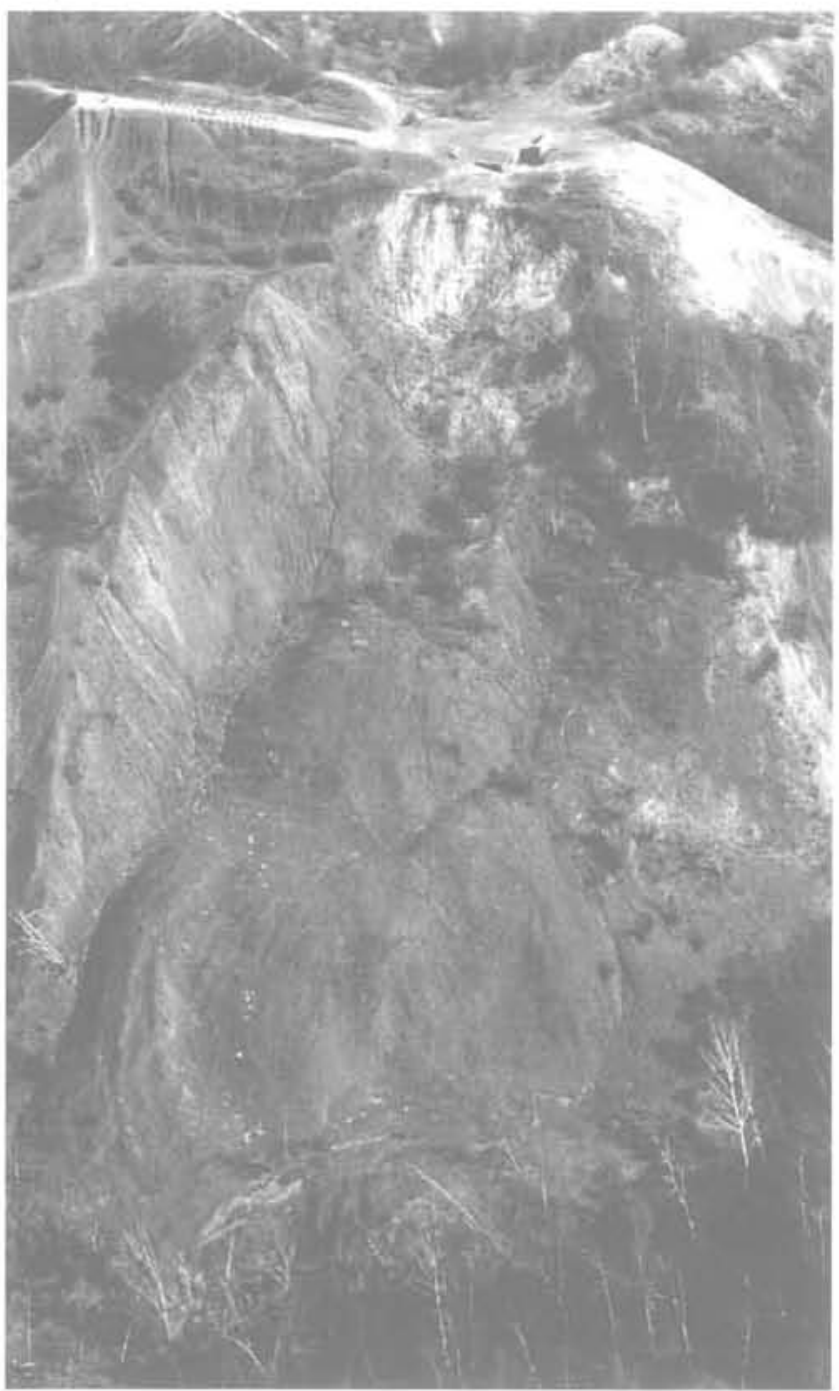

FG. 19 Glissement Ouest. En bas de l'image, les terrains glissés, en haut, en clair (rouge), le plan de glissement (discontinuité entre les schistes brulés et non brûlés).

West slide. At the bottorn of the picture, the flooded soils. At the top, the light (red) zone corresponds to the failure surface (discontinuity between the $\alpha$ red $n$ and $\propto$ black $n$ schists.

relevé du mouvement et des désordres ainsi qu'un lever topographique et que l'examen d'anciennes cartes topographiques et photos aériennes, les reconnaissances ont consisté en une investigation du soussol effectuée par:

- une prospection géophysique (sismique réfraction et résistivité électrique) réalisée en périphérie du terril, sur le flanc du terril et au pied du glissement Ouest. La prospection géophysique a pour but de déterminer la structure générale du site et de permettre l'implantation judicieuse des reconnaissances mécaniques ;

- neuf petits forages d'étalonnage réalisés à la tarière hélicoïdale sur le pourtour du terril avec prélèvements d'échantillons non remaniés pour essais de laboratoire;

- sept essais de pénétration statique 10T implantés en fonction des résultats de la prospection géophysique; - huit essais de cisaillement direct réalisés sur les échantillons prélevés dans les forages.

La prospection géophysique par sismique réfraction permet de distinguer trois niveaux de terrains caracté- risés par les vitesses apparentes de propagation des ondes sismiques de compression (V) :

- terrains superficiels : terre végétale, remblais de terre et cailloux, schistes du terril, limons sableux/argileux quaternaire (V = 130 à $240 \mathrm{~m} / \mathrm{s}$ ), sable yprésien et landénien hors nappe $(\mathrm{V}=260$ à $550 \mathrm{~m} / \mathrm{s})$ et terril plus compact $(\mathrm{V}=700$ à $710 \mathrm{~m} / \mathrm{s})$;

- schistes du terril sous nappe (V variant avec le degré de compacité de 1220 à $1600 \mathrm{~m} / \mathrm{s}$ ) et limons sableux/argileux quaternaires sous nappe $(\mathrm{V}=1365$ à $1575 \mathrm{~m} / \mathrm{s}$ )

- argiles (sableuses) yprésiennes sous nappe ( $\mathrm{V}=1740$ à $2515 \mathrm{~m} / \mathrm{s})$ ou le sable landénien sous eau $(\mathrm{V}=1580$ à $1860 \mathrm{~m} / \mathrm{s}$ ).

Les sondages électriques distinguent les formations suivantes:

- terrains superficiels : remblais de terre et cailloux. résistivité $=300 \Omega$.m:

- limons sableux/argileux quaternaires : résistivité $=50$ à $65 \Omega$.m non saturés et $=13$ à $20 \Omega$.m sous nappe ; - formations sableuses yprésiennes (résistivité $=35 \Omega \mathrm{m}$ sous nappe) ;

- formations sableuses landéniennes (résistivité = $60 \Omega . m$ non saturé et $35 \Omega . m$ sous nappe) ;

- argile yprésienne : résistivité $=10$ à $15 \Omega . \mathrm{m}$.

Les essais de pénétration statique, implantés en fonction des résultats de la prospection géophysique, ont permis de mettre en évidence trois couches de terrains dont les caractéristiques géomécaniques sont les suivantes :

- schistes du terril caractérisés par une résistance à la pointe de 40 à $50 \mathrm{~kg} / \mathrm{cm}^{2}$ mais présentant des « pics $)$ de résistance à la pointe assez importants $\left(>180 \mathrm{~kg} / \mathrm{cm}^{2}\right)$. Ces pics sont accompagnés de chocs lors de l'enfoncement du pénétromètre;

- silts sableux dont les caractéristiques sont assez similaires à celles des schistes du terril mais ne présentant pas les mêmes \& pics » de résistance à la pointe ni les chocs. La résistance moyenne de ces silts sableux est de 30 à $40 \mathrm{~kg} / \mathrm{cm}^{2}$ avec des passages plus résistants correspondant aux passages plus sableux :

- argile et silts argileux caractérisés par une résistance à la pointe augmentant régulièrement avec la profondeur d'investigation. Le frottement latéral augmentant environ de 300 à $700 \mathrm{~kg} / \mathrm{m}$ de profondeur.

Les forages complémentaires ont fourni les échantillons non remaniés pour les essais de laboratoire et ont permis d'étalonner les autres méthodes. Sur base des reconnaissances, on a pu tracer les coupes géologiques interprétatives dont la figure 20 donne un exemple avec, sous les remblais du terril, la succession des couches du Tertiaire, avec un léger pendage nord.

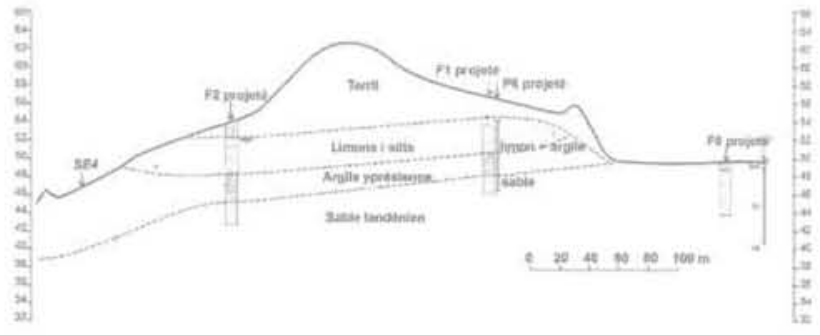

FG. 20 Coupe géologique nord-sud.

North-South geological cross - section.

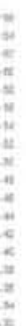


Les résultats des essais de laboratoire, essais de cisaillement direct consolidés-drainés, réalisés sur les échantillons remaniés d'argile et de limons prélevés dans les forages (les « limons » désignent les formations silto-sableuses surmontant les formations yprésiennes et les " argiles $»$ désignent ces formations yprésiennes argileuses) donnent globalement les caractéristiques mécaniques suivantes :

- Limons

$\gamma_{\mathrm{d}}$ : de 10,75 à $15,89 \mathrm{kN} / \mathrm{m}^{3}$,

w naturelle : de 25,1 à $57,2 \%$,

au pic : $\varphi^{\prime}$ de 18,1 à $25^{\circ}, c^{\prime}$ de 5 à $10 \mathrm{kPa}$,

en résiduelle : $\varphi^{\prime}$ de 15,7 à $22,5^{\circ} \mathrm{C}^{\prime}$ de 0 à $10 \mathrm{kPa}(0$ à $\left.1,0 \mathrm{t} / \mathrm{m}^{2}\right)$.

- Argiles :

$\gamma_{\mathrm{d}}:$ de 12,51 à $14,87 \mathrm{kN} / \mathrm{m}^{3}$,

w naturelle : de 28,04 à 41,7\%,

au pic : $\varphi^{\prime}$ de 17,3 à $21,6^{\circ} \mathrm{C}^{\prime}$ : de 8 à $18 \mathrm{kPa}$,

en résiduelle : $\varphi^{\prime}$ : de 14 à $20,5^{\circ} \mathrm{C}^{\prime}$ de 4,7 à $12,8 \mathrm{kPa}$.

Avec ces valeurs, recalibrées au cours de l'étude par back-analysis, les profils des glissements Est et Ouest ont été étudiés au point de vue stabilité. Les calculs ont été réalisés par la méthode de Janbu au moyen d'un programme développé aux LGIH, permettant d'inclure les effets de surpressions interstitielles et les effets sismiques (en calcul statique).

\section{3}

\section{Étude de stabilité - Glissement Est}

La structure des terrains et des courbes de glissement étudiées au profil Est est donnée à la figure 21. Sur cette figure sont représentées quelques courbes de glissement étudiées et quelques hypothèses sur le niveau de la nappe du terril.

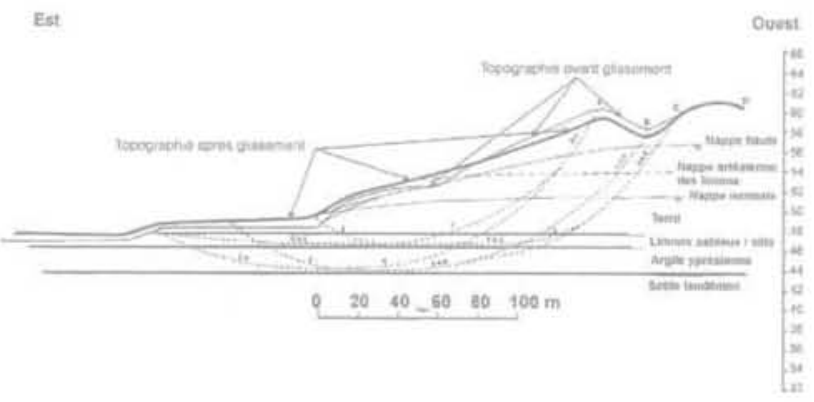

FG.21 Coupe dans le glissement Est : exemples de courbes de glissement.

Cross section in the East slide: examples of rupture lines.

Le calcul de la stabilité de ce profil a été réalisé en deux étapes : en considérant la topographie avant glissement et dans la situation topographique actuelle. Avant glissement, avec les paramètres mécaniques donnés plus haut et une position basse de la nappe phréatique, les coefficients de sécurité obtenus sont supérieurs à 1,25 , ce qui est une valeur faible mais cependant supérieure à l'unité, valeur incompatible avec le fait qu'un glissement s'est effectivement pro- duit. Une étude paramétrique a permis de restituer (par back-analysis) les conditions dans lesquelles le glissement a pu se produire. Les variations des paramètres mécaniques (sauf pour l'argile yprésienne) ont relativement peu d'importance par rapport à celles de la nappe phréatique.

En effet, en cas d'augmentation du niveau piézométrique de la nappe, les coefficients de sécurité décroissent fortement, tendant à se rapprocher de l'instabilité (valeurs proches de l'unité ou inférieures). La probabilité d'une nappe aussi haute est faible. Les causes de l'instabilité doivent être cherchées dans d'autres actions de la nappe que la simple élévation du niveau piézométrique. Il semble que le poids du terril et du bourrelet Est ait diminué la perméabilité des limons, ce qui a confiné la nappe à l'exutoire. Au cours de longues périodes de précipitation, il y a donc possibilité d'avoir une nappe captive (artésienne) dans les sables et silts sous les limons quaternaires compactés par le terril. Il s'avère que la présence d'une nappe artésienne, associée à une légère diminution des caractéristiques de l'argile yprésienne, peut expliquer le glissement observé.

Avec les paramètres ainsi déduits de l'étude du glissement, il a été possible de définir les orientations des solutions de confortement possible : drainage ou stabilisation par risberme. La solution retenue et dimensionnée par le bureau d'études Tractebel comprend la construction d'une risberme en pied de glissement, avec drainage approprié. Les travaux de confortement ont été terminés dans le courant 2000.

\section{4}

\section{Étude de stabilité - Glissement Ouest}

Le glissement Ouest s'est produit dans la partie supérieure du terril, entre les cotes 100 et 130, provoquant une sorte de coulée boueuse qui s'est déposée en contrebas, sous la cote 85 , renversant les arbres et provoquant quelques bourrelets supplémentaires en pied de terril. Entre les cotes 85 et 100, la topographie est restée intacte. Le plan de glissement laisse apparaître le schiste rouge brûlé. La coulée de schiste en contrebas ne montre pratiquement pas de schistes brûlés. Il est donc probable que le plan de glissement coincide avec le contact schistes brûlés chauds/schistes non brûlés. Les figures 22 et 23 présentent respectivement une coupe complète du versant au droit du glissement Ouest et un agrandissement de la partie supérieure du versant, c'est-à-dire le glissement proprement dit.

L'examen de la topographie actuelle montre également que le glissement s'est produit en deux phases: un premier glissement s'est déclenché suivant la ligne de glissement CG1 (entre les points A et B à la figure 23) à l'endroit oủ le terril montrait la plus forte pente. Le premier glissement a provoqué la déstabilisation de la partie supérieure et un deuxième glissement s'est produit suivant la ligne de glissement CG2 (entre les points C et D à la figure 23). Cette partie du terril était stable depuis de nombreuses années. Le glissement (le double glissement) s'est produit en été, lors de fortes pluies. Le mécanisme du glissement est donc tout à fait en conformité avec ce qui a été décrit plus haut, à savoir, les effets combinés de l'eau saturant le schiste $\alpha$ noir $)$, la surface de discontinuité entre les 


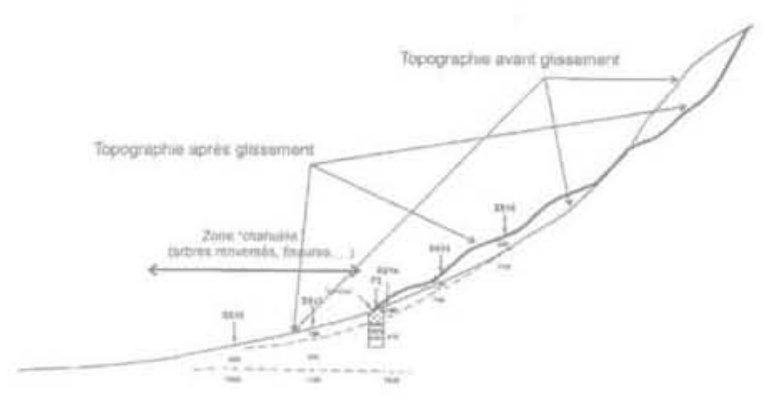

Q $\quad 20 \quad 40 \quad 60 \quad 80 \quad 100 \mathrm{~m}$

FG. 22 Coupe d'ensemble dans le versant du glissement Ouest.

General cross section of the slope of the West slide.

schistes $\alpha$ noirs $)$ et $\alpha$ rouges $»$ et la pression de vapeur s'exerçant en partie haute du terril.

L'analyse de la situation avant glissement a été faite avec les valeurs suivantes des paramètres physiques et mécaniques du terril, choisies en fonction des données d'études précédentes :

- poids volumique $\mathrm{sec}$ : $19 \mathrm{kN} / \mathrm{m}^{3}$;

- angle de frottement interne : $35^{\circ}$;

- cohésion (tenant compte que cette zone du terril est peu compactée) : $5 \mathrm{kPa}$.

La nappe est supposée plus basse que la courbe de glissement. Dans ces hypothèses, les coefficients de sécurité obtenus sont supérieurs ou égaux à 1,5. Le terril est donc stable mais sans sécurité totalement satisfaisante. Dans ces conditions, il est normal qu'il ait tenu depuis sa construction.

Dans la situation actuelle, en considérant les paramètres obtenus par l'analyse du glissement, en particulier les hypothèses sur les effets de l'eau, les coefficients de sécurité déterminés dans les hypothèses d'un terrain à humidité naturelle $(9 \%)$ puis complètement saturé en cas d'orages violents, avec une pression interstitielle et une sous-pression de vapeur, les coefficients de sécurité descendent en dessous de l'unité. En cas de forts orages, tels ceux qui ont provoqué le glissement de ce flanc, l'instabilité est pratiquement inévitable. La seule solution envisageable pour conforter le flanc Ouest a été de reprofiler la partie haute du terril pour lui donner une pente plus en rapport avec les caractéristiques mécaniques de ce type de terrains. Ce reprofilage a été dimensionné par le bureau d'études Tractebel et est actuellement réalisé.

\section{7}

\section{Conclusion}

Dans les études de terrils, aussi bien ceux en place que ceux en cours de remodelage, interviennent de nombreux facteurs, naturels ou anthropiques. Les quelques exemples présentés montrent l'importance de

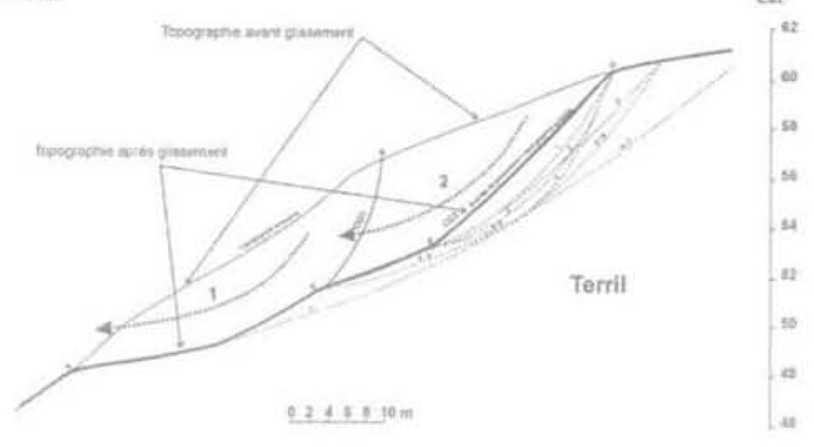

FG. 23 Détail du glissement Ouest : décomposition des mouvements.

Detail of the West slide : succession of motions.

l'effet de la combustion interne dans les problèmes d'instabilités de terrils. Lorsque de tels problèmes se posent, il est nécessaire d'étudier les solutions en gardant présent à l'esprit le fait qu'il n'est pratiquement pas possible d'éteindre un terril en combustion (de nombreuses tentatives ont été faites sans succès). Il faut aussi s'assurer que des interventions (comme une excavation) ne risqueront pas de provoquer d'explosion, comme cela s'est déjà produit.

Dans tous les cas, une étude détaillée est indispensable pour déterminer l'ampleur du risque potentiel. Cette étude comporte dans tous les cas une prospection géophysique sismique et électrique, les tomographies électriques permettant, sur base des résistivités, de déterminer les zones en combustion, brûlées ou non brûlées et la prospection sismique donnant les épaisseurs et les compacités relatives des différents terrains. On peut ainsi déterminer les zones où le schéma de glissement tel qu'il a été décrit est susceptible de se produire et définir les zones à risques. Les informations fournies par les termographies infrarouges complètent le dispositif et permettent un suivi assez aisé de l'évolution de la combustion.

Dans le cas le plus général, ces reconnaissances géophysiques sont complétées d'une campagne de reconnaissance classique comportant des essais mécaniques in situ, des prélèvements d'échantillons et des essais de laboratoire permettant de réaliser les études de stabilité conduisant à la préconisation des solutions à adopter. Ces solutions sont à déterminer au cas par cas, en tenant compte de tous les facteurs, aussi bien géotechniques qu'environnementaux, économiques et sociaux.

\section{REMERCIEMENTS}

Les auteurs tiennent à remercier les administrations communales de Herstal Charleroi et Mons et en particulier Madame Rouhart, directrice de l'Urbanisme de la ville de Mons, Monsieur Monseux, échevin de la ville de Charleroi et Madame Piret, du service Environnement de la ville de Charleroi pour leur aide et l'intérêt particulier qu'ils ont porté à tous les aspects de ces études. Ils tiennent à signaler que les photos infrarouges ont été réalisées par des vols d'hélicoptères de la gendarmerié. 
Carras J.N., Leventhal A.R. - $\alpha$ Spontaneous combustion in open-cut coal mining spoil piles : a challenging legacy \#. Proc. Geo, Eng. Melbourne. 2000 , p. 313.

Collectif - Spoil heaps and lagoons, London: Ed. National Coal Board, 1970

Fredlund D.G.. Rahardjo H. - Soil mechanics for unsaturated soils. John Wiley \& Sons Ed., 1993

Glasser D., Bradshaw S, - a Spontaneous combustion in beds of coal $\mathrm{x}$. In : Handbook of Heatand Mass Transfer, vol. 4 Advances in Reactor Design and Com bustion Science, 1990, p. 1071-1148, Gulf. N. Cheremisinoff, Ed.
Harrington D., Heast J.H. - $\alpha$ Incendies des terris 3. Annales des Mines de Belgique XLVIII (4), 1949, p. 427-434.

Hebley H.F. - $\alpha$ La lutte contre les feux de terril 10, traduit par J. Lecomte. Annales des Mines de Belgique XLVIII (6), 1949. p. 709-712.

Lohest M. - a Soufre sur le terril en combustion du charbonnage de Werister 1. Annales de la Société Géologique de Belgique vol. 31, 1903, p. 128-132.

Linard de Guertechain A. - is Action tectonique des terrils. Glissements de Courcelles et de Fontaine-l'Evêque 1)، Bull Tec. Union des étudiants sortis des Écoles spéciales de Louvain 1939, p.39-61.
Marlière R. - $\alpha$ Terrils $\alpha$ en marche nn is. Bull. A.I.Ms. $n^{\circ} 4,1950$, p. 11-18.

Monjole, A. - $\alpha$ Les friches de charbon de la région liégeoise m. Bull. Soc. Royale des sciences de Liège, vol. 69. 5, 2000 .

Schroeder Ch. - « Exploitation rationnelle dun terril o. Revue Française de Géotechnique $n^{\circ} 14$ bis * Environnement et Géotechnique v, 1981, p. 80-84.

Wathelet M. - "Terril de I'Héribus Etudes des conditions géologiques et géotechniques .. Travail de fin d'études Ulg. Facuité des Sciences appliquées. 1997. 